\title{
Análise dos óbitos em idosos no Estudo SABE*
}

\author{
ANALYSIS OF DEATHS OF ELDERLY PEOPLE IN THE SABE SURVEY
}

ANÁLISIS DE LAMUERTE DE ANCIANOS EN EL ESTUDIO SABE

Flávia de Oliveira Motta Maia'1, Yeda Aparecida de Oliveira Duarte², Maria Lúcia Lebrão³

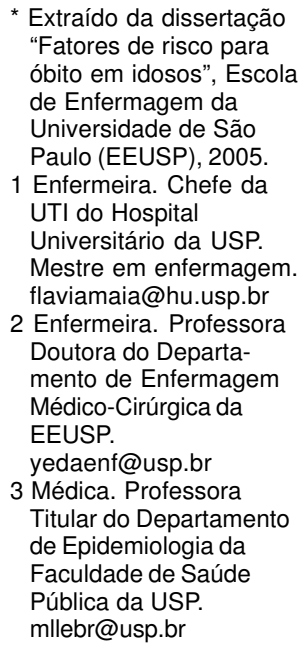

\section{RESUMO}

O Estudo SABE - Saúde, Bem

Estar e Envelhecimento - foi coordenado pela Organização Pan-Americana de Saúde, envolvendo sete países da América Latina e Caribe. No Brasil, foi desenvolvido no município de São Paulo, em 2000. Para a amostra de 2.143 idosos, foi aplicado um instrumento, composto por onze seções, em duas fases. $\mathrm{O}$ presente estudo teve por objetivo caracterizar e analisar as causas de óbito dos idosos, durante o período da coleta dos dados do SABE. Do total de óbitos ocorridos, $55,3 \%$ pertenciam ao sexo masculino e a idades avançadas. A maioria dos idosos vivia acompanhada e não precisou de ajuda para responder ao questionário. As doenças que apresentaram maior incidência foram: aparelho circulatório, neoplasias e aparelho respiratório. Espera-se que os resultados desse estudo possam contribuir para a adoção de medidas preventivas que visem à diminuição dos riscos relacionados aos óbitos entre idosos.

\section{DESCRITORES}

Idoso.

Mortalidade.

Morbidade.

\section{ABSTRACT}

The Pan-American Health Organization coordinated a survey called SABE - acronym for Health, Wellbeing and Aging carried out in seven Latin America and Caribbean countries. In Brazil, it was developed in the city of São Paulo in 2000. On a sample of 2,143 elderly people was applied an instrument, made up of eleven sections, in two parts. The present study was aimed at characterizing and analyzing the death causes of the elderly people during the period of the SABE data collection. From the total deaths, $55.3 \%$ were of very old males. Most elderly people didn't live alone and needed no help in answering the questionnaire. Regarding death causes, the diseases that had most incidences were circulatory system, neoplasias and respiratory system. It is hoped that the results of this study may contribute to the adoption of preventive measures aimed at reducing risks related to death among elderly people.

\section{KEY WORDS \\ Aged. \\ Mortality. \\ Morbidity.}

\section{RESUMEN}

El Estudio SABE - Salud, Bienestar y Envejecimiento - fue coordinado por la Organización Panamericana de la Salud, involucrando a siete países de América Latina y el Caribe. En el Brasil, fue desarrollado en el municipio de Sao Paulo, en el 2000. Para la muestra de 2,143 ancianos, se aplicó un instrumento, compuesto por once secciones, en dos fases. En el presente estudio se tuvo por objetivo caracterizar y analizar las causas de muerte de los ancianos, durante el período de la recolección de los datos del SABE. Del total de muertes ocurridas, el 55,3\% pertenecía al sexo masculino y a edades avanzadas. La mayoría de los ancianos vivía acompañado y no necesitó de ayuda para responder el cuestionario. Las enfermedades que presentaron mayor incidencia fueron del aparato circulatorio, neoplasias y aparato respiratorio. Se espera que los resultados del estudio contribuyan a la adopción de medidas preventivas que visen la disminución de los riesgos relacionados a la muerte de los ancianos.

\section{DESCRIPTORES}

Anciano.

Mortalidad.

Morbidad. 


\section{INTRODUÇÃO}

Envelhecer é um processo natural e fisiológico que se caracteriza pela diminuição progressiva da reserva funcional orgânica, o que ocasiona uma maior dificuldade na manutenção do equilíbrio homeostático quando o idoso é exposto a situações de sobrecarga, tornando-o, assim, mais susceptível a agravos e doenças, principalmente as de natureza crônico-degenerativas, que podem culminar com a morte $^{(1-2)}$.

O perfil de morbi-mortalidade do Brasil está sendo alterado pelo envelhecimento populacional, fazendo com que o cenário caracterizado por uma população jovem, com maior incidência de doenças infecciosas, transforme-se em um outro, típico de uma população mais envelhecida, onde predominam agravos crônicos e o conseqüente aumento nos custos assistenciais, gerados pelas alterações na utilização dos serviços de saúde, tais como: aumento das hospitalizações e necessidade de incremento de serviços de reabilitação ${ }^{(3-5)}$.

A prevalência de doenças crônicas na população idosa, no Município de São Paulo, mostrou-se elevada, ou seja, houve o relato de mais de três doenças concomitantes em $48 \%$ das mulheres e em $33 \%$ dos homens ${ }^{(6)}$. Ainda segundo esse estudo, as principais doenças crônicas referidas pelos idosos foram: hipertensão $(53,3 \%)$, doenças ósteo-articulares $(31,7 \%)$, problemas cardíacos $(19,5 \%)$, diabetes $(17,9 \%)$, osteoporose $(14,2 \%)$, doenças crônicas pulmonares $(12,2 \%)$, doenças circulatórias $(7,2 \%)$ e neoplasias malignas $(3,3 \%)$.

A análise das principais causas de óbito entre os idosos brasileiros, no ano 2002, identifica as doenças do aparelho circulatório $(36,0 \%)$, as neoplasias $(14,7 \%)$ e as doenças do aparelho respiratório $(12,6 \%)$ como as mais importantes, correspondendo, somente essas três, a mais de $60 \%$ do total de óbitos ${ }^{(7)}$. As doenças cerebrovasculares e isquêmicas do coração têm predomínio entre as doenças do aparelho circulatório. As neoplasias malignas da traquéia, dos brônquios e dos pulmões têm maior frequiência para ambos os sexos. Para homens, a neoplasia de próstata e, para mulheres, a neoplasia da mama, constituem a segunda causa de óbito entre os idosos. As doenças do aparelho respiratório predominantes são a doença pulmonar obstrutiva crônica (DPOC), a pneumonia e a influenza para ambos os $\operatorname{sexos}^{(8)}$.

Frente ao panorama observado, verifica-se a necessidade urgente de desenvolvimento de políticas públicas adequadas, com ênfase em estruturas sociais e assistência à saúde que atendam às demandas específicas para essa faixa etária, a fim de minimizar as incapacidades e complicações causadas por doenças que interferem diretamente no desempenho das atividades cotidianas dos idosos, principalmente as condições crônicas que não são adequadamente controladas. A prevenção de doenças crônicas e suas seqüelas é possível pelo controle de suas causas ou fatores de risco, o que pode ser obtido pelo incremento dos programas de prevenção de doenças crônicas já implementados.

\section{O ESTUDO SABE}

A Organização Pan-Americana de Saúde coordenou um estudo multicêntrico denominado Saúde, Bem-Estar e Envelhecimento - SABE, para traçar o perfil dos idosos na América Latina e no Caribe. Participaram desse estudo a Argentina, Barbados, Brasil, Chile, Cuba, México e Uruguai. No Brasil, a população do estudo foi composta pelos idosos residentes, no ano 2000, no Município de São Pau$10^{(9)}$. A amostra final, de 2.143 idosos, foi composta pela somatória de uma amostra probabilística (sorteio) e de uma amostra intencional (composição livre para os grupos ampliados para complementar a amostra de idosos em velhice avançada) $)^{(10)}$.

Os dados foram colhidos simultaneamente, por meio de entrevistas domiciliares, feitas com um instrumento constituído por onze blocos temáticos ${ }^{(a)}$. A coleta de dados ocorreu em duas etapas, com um período máximo de seis meses entre elas. Na primeira, foram preenchidas as seções de A a J (questionário) e, na segunda, as seções K e L, constituídas da antropometria e testes de mobilidade e flexibilidade. Durante esse intervalo foram evidenciados 38 óbitos, o que motivou o desenvolvimento do presente estudo.

\section{OBJETIVO}

Caracterizar e analisar as causas de óbito dos idosos residentes no Município de São Paulo e que faleceram entre a primeira e a segunda etapas de coleta de dados do Estudo SABE.

\section{CASUÍSTICA E MÉTODO}

Este trabalho é parte do Estudo SABE e caracterizou-se como uma pesquisa exploratória, descritiva, aplicada, de campo, retrospectiva, com abordagem quantitativa.

A população de estudo foi composta por 38 idosos que participaram da primeira etapa e que tinham ido a óbito quando da realização da segunda etapa, que ocorreu no período máximo de seis meses.

A caracterização dos óbitos foi realizada inicialmente traçando o perfil dos idosos que morreram no período, utilizando-se dados sócio-demográficos (como sexo, idade, residindo só ou acompanhado) e a presença ou não de um proxi-

(a) Disponível em: http://www.fsp.usp.br/sabe 
respondente ${ }^{(\mathrm{b})}$, por meio dos dados constantes da Seção A informações pessoais. Para a análise comparativa das informações fornecidas pelo idoso quanto ao seu estado de saúde, na primeira fase da coleta de dados e a causa de óbito, foram utilizadas as questões da seção $\mathrm{C}$, estado de saúde do Questionário SABE.

Os dados referentes aos óbitos foram extraídos dos Bancos de Dados de Registro de Óbito do Estado e do Município de São Paulo, dos anos 2000 e 2001, fornecidos pela Fundação Sistema Estadual de Análise de Dados (SEADE) e pelo Programa de Aprimoramento das Informações de Mortalidade no Município de São Paulo (PRO-AIM). Nesses bancos de dados, foram procurados, pelos nomes, os 38 casos de óbitos dos idosos que participaram do Estudo SABE. Quando identificados, os dados do registro de óbito eram comparados aos dados constantes na Seção A, infor- mações pessoais do questionário, de forma a evitar a ocorrência de homônimos. Após a identificação e a comparação, foram extraídos do Registro de Óbito as informações referentes à causa básica e às secundárias.

O Estudo SABE foi submetido e aprovado pelo Comitê de Ética em Pesquisa da Faculdade de Saúde Pública (Parecer COEP 67/99) e pelo Conselho Nacional de Ética em Pesquisa (Parecer CONEP 315/99).

\section{RESULTADOS E DISCUSSÃO}

Todos os óbitos do estudo foram identificados nos Bancos de Dados de Registro de Óbito do Estado e do Município de São Paulo, dos anos 2000 e 2001. As Tabelas 1 e 2 mostram a caracterização desses idosos.

Tabela 1 - Número e proporção (\%) de óbitos segundo sexo e idade, Estudo SABE - São Paulo - 2000

\begin{tabular}{|c|c|c|c|c|c|c|}
\hline \multirow{2}{*}{$\begin{array}{l}\text { Idade (em } \\
\text { anos) }\end{array}$} & \multicolumn{2}{|c|}{ Feminino } & \multicolumn{2}{|c|}{ Masculino } & \multicolumn{2}{|c|}{ Total } \\
\hline & $\mathbf{n}$ & $\%$ & $\mathbf{n}$ & $\%$ & $\mathbf{n}$ & $\%$ \\
\hline 60 a 69 & 1 & 5,9 & 3 & 14,3 & 4 & 10,5 \\
\hline 70 a 79 & 5 & 29,4 & 8 & 38,1 & 13 & 34,2 \\
\hline 80 e mais & 11 & 64,7 & 10 & 47,6 & 21 & 55,3 \\
\hline Total & 17 & 100,0 & 21 & 100,0 & 38 & 100,0 \\
\hline
\end{tabular}

Tabela 2 - Número e proporção (\%) de óbitos segundo presença de acompanhante e ajuda para responder ao questionário, Estudo SABE - São Paulo - 2000

\begin{tabular}{lrc}
\hline Presença de acompanhante & $\mathbf{n}$ & $\%$ \\
Vive acompanhado & 32 & 84,2 \\
Vive sozinho & 6 & 15,8 \\
\hline Ajuda para responder o questionário & $\mathbf{n}$ & $\%$ \\
Com ajuda & 31 & 81,6 \\
Sem ajuda & 7 & 18,4 \\
\hline Total & 38 & 100,0 \\
\hline
\end{tabular}

Observa-se, na análise dos óbitos, a ocorrência de maior mortalidade entre os homens, corroborando as estatísticas nacionais de óbito. Os homens, em todas as faixas etárias, apresentam maior mortalidade em relação às mulheres, pela maior exposição a riscos ambientais e sociais. Entre os idosos, essa diferença torna-se ainda mais acentuada, ocasionando o fenômeno denominado feminização da velhice. Quanto mais envelhecida a população, maior será a proporção de mulheres em relação aos homens da mesma faixa etária $^{(11)}$.

(b) Proxi-respondente é a pessoa que auxilia o entrevistado, na sua impossibilidade, por dificuldades físicas ou cognitivas, a responder o questionário.
A análise de sobrevida dos idosos sem incapacidade mostrou que as mulheres sobrevivem mais que os homens, porém com maiores índices de incapacidade ${ }^{(12)}$. Esses dados, associados aos encontrados por em outro estudo ${ }^{(13)}$, mostram uma proporção significativamente menor de auxílio no desempenho das atividades de vida diária para as mulheres do que para os homens.

Desta forma, o envelhecimento feminino parece ocorrer com maior sofrimento, exigindo atenção especial na elaboração de políticas públicas que atendam às demandas específicas dessa população, quer no auxílio a um envelhecimento feminino com melhor qualidade, quer na prevenção dos óbitos dos homens idosos.

Em relação à estratificação etária, observa-se um aumento dos óbitos em idades mais avançadas. Essa relação é esperada, uma vez que, quanto maior a idade, maior também é a probabilidade que o indivíduo tem de morrer, pois, com o passar dos anos, há uma diminuição da reserva funcional, o que torna as pessoas mais suscetíveis a doenças e eventos fatais ${ }^{(2)}$. O que se espera, no entanto, é que os eventos fatais entre os idosos em velhice avançada não sejam precedidos de longos períodos de doenças, incapacidade e sofrimento. 
Os grandes esforços na área de Gerontologia visam a postergar ao máximo a morbidade, ou seja, contribuir para que as pessoas vivam mais tempo, livres de doenças, ou com as mesmas controladas a ponto de não gerarem incapacidade. A vida, num determinado momento, culminará em um evento fatal, porém de forma mais repentina, preferencialmente com menor ou sem sofrimento e em idade bem $\operatorname{avançada}^{(3,14-15)}$.

Dentro desses pressupostos, a capacidade funcional passa a ser um novo paradigma de saúde para o envelhecimento pois, por meio da sua manutenção, o idoso, mesmo apresentando doenças crônicas, pode ser considerado saudável e com condições de manter sua independência e autonomia, fatores indispensáveis para se obter boa qualidade de $\operatorname{vida}^{(3,14-15)}$.

Neste estudo, verificou-se que, dos idosos que morreram, a maioria vivia acompanhada. Esse resultado reflete a população de idosos do Município de São Paulo, pois a maioria (aproximadamente $87 \%$ ) mora com outras pessoas, familiares ou não. Tal achado pode indicar uma condição de maior proteção, uma vez que, conforme estudo ${ }^{(16)}$ a co-residência representa uma condição de maior proteção ao idoso pois, nessas circunstâncias, haveria maior potencial de auxílio disponível para assistir às diferentes demandas apresentadas por eles. Poderia, também, por outro lado, indicar uma condição de maior fragilidade, visto que alguns deles, possivelmente, não teriam condições de morar sozinhos, quer pelas doenças apresentadas, quer pelas limitações funcionais que poderiam existir em decorrência delas.

Outra característica dos idosos que morreram é que a maioria $(81,6 \%)$ foi capaz de responder ao questionário SABE sem ajuda, o que indica uma boa preservação da condição cognitiva e o não comprometimento da qualidade da informação. De certa forma, esse fato reforça a afirmação anterior sobre a necessidade de gerenciamento adequado e contínuo da condição de saúde dos idosos, em especial os mais fragilizados, pois os agravos e os quadros de doenças podem modificar-se repentinamente e evoluir para eventos fatais.

Buscando compreender as razões que levaram esses idosos a morrer, as causas de óbito foram classificadas e distribuídas segundo a Classificação Internacional de Doenças $10^{\text {a }}$ revisão (CID-10) ${ }^{(17)}$ e sexo (Tabela 3 ).

Tabela 3 - Número e percentagem de óbitos segundo causa básica e sexo, Estudo SABE - São Paulo - 2000

\begin{tabular}{|c|c|c|c|c|c|c|}
\hline \multirow{2}{*}{ Causa básica de óbito (CID-10) } & \multicolumn{4}{|c|}{ Sexo } & \multicolumn{2}{|c|}{ Total } \\
\hline & $\mathbf{n}$ & $\%$ & $\mathrm{n}$ & $\%$ & $\mathbf{n}$ & $\%$ \\
\hline Doenças do aparelho circulatório & 9 & 52,9 & 9 & 42,9 & 18 & 47,4 \\
\hline Neoplasias malignas & 1 & 5,9 & 5 & 23,8 & 6 & 15,8 \\
\hline Doenças do aparelho respiratório & 1 & 5,9 & 4 & 19,0 & 5 & 13,1 \\
\hline Doenças do aparelho digestivo & 2 & 11,8 & 1 & 4,8 & 3 & 7,9 \\
\hline Doenças endócrinas, nutricionais e metabólicas & 1 & 5,9 & 2 & 9,6 & 3 & 7,9 \\
\hline Outras causas & 3 & 17,6 & - & - & 3 & 7,9 \\
\hline Total & 17 & 100,0 & 21 & 100,0 & 38 & 100,0 \\
\hline
\end{tabular}

Verifica-se que os principais grupos de causas de óbito entre os idosos neste estudo coincidem com as estatísticas nacionais. As doenças do aparelho circulatório representaram quase metade do total dos óbitos (47\%). As taxas de mortalidade por doenças cardiovasculares aumentam conforme o avançar da idade. A tabela 4 mostra as causas desses óbitos de forma detalhada.

Na maioria dos países do mundo, a principal causa de morte por doenças do aparelho circulatório é a cardiopatia coronariana, seguida pelo acidente cerebrovascular, tanto para homens quanto para mulheres. Outras doenças cardiovasculares de prevalência importante em idosos são a hipertensão, a insuficiência cardíaca e a demência vascular ${ }^{(18)}$.

Segundo pesquisadores brasileiros ${ }^{(6)}$, vários estudos longitudinais e transversais confirmam que a hipertensão é um importante fator preditivo de mortalidade e morbidade cardiovascular entre os idosos. A pressão arterial elevada é comum nos idosos, chegando a aproximadamente $50 \%$ de incidência, com variações nas diversas regiões do mundo ${ }^{(18)}$.

A insuficiência cardíaca atinge cerca de $1 \%$ das pessoas acima dos 65 anos, ocorrendo na mesma freqüência para homens e mulheres. Dentre os que apresentam essa doença, apenas $15 \%$ continuam vivos após 10 anos do diagnóstico, sendo que os homens sobrevivem menos que as mulheres $^{(18)}$.

A origem vascular, em muitos países, é a segunda causa de demência, perdendo apenas para a Doença de Alzheimer. Quanto maior a idade, maior a incidência da demência vascular, chegando a representar 1/3 a 1/4 do total de casos de demência grave ${ }^{(18)}$. 
Tabela 4 - Número e proporção (\%) de óbitos segundo causas básicas, Estudo SABE - São Paulo - 2000

\begin{tabular}{lrr}
\hline Causa básica do óbito segundo a CID-10 & $\mathrm{n}$ & $\%$ \\
\hline Doenças do aparelho circulatório & 18 & 47,4 \\
Doenças hipertensivas & 2 & 5,3 \\
Doenças isquêmicas do coração & 5 & 13,2 \\
Outras formas de doença do coração & 4 & 10,5 \\
Doenças cerebrovasculares & 7 & 18,4 \\
Neoplasias malignas & 6 & 15,8 \\
Lábio, cavidade oral e faringe & 1 & 2,6 \\
Órgãos digestivos & 1 & 2,6 \\
Aparelho respiratório & 2,6 \\
Trato urinário & 1 & 2,6 \\
Glândulas endócrinas & 1 & 2,6 \\
Tecido linfático e hemotopoético & 2,6 \\
Doenças do aparelho respiratório & 1 & 13,2 \\
Gripe eneumonia & 1 & 5,3 \\
Doenças crônicas das vias aéreas inferiores & 5 & 7,9 \\
Doenças do aparelho digestivo & 2 & 7,9 \\
Esôfago, estômago e duodeno & 3 & 2,6 \\
Hérnia & 3 & 2,6 \\
Outras doenças dos intestinos & 1 & 2,6 \\
Doenças endócrinas, nutricionais ou metabólicas & 1 & 7,9 \\
Diabetes & 1 & 2,6 \\
Desnutrição & 3 & 2,6 \\
Distúrbios metabólicos & 1 & 2,6 \\
Outras causas & 1 & 7,9 \\
Outros transtornos do sistema nervoso & 1,6 \\
Outras doenças do aparelho urinário & 3,6 \\
Outras doenças da pele e do tecido subcutâneo & 3 & 2,6 \\
Total & 1 & $100,0 *$ \\
\hline
\end{tabular}

* Obs: houve arredondamento das percentagens.

Fonte: Fundação SEADE e PRO-AIM.

Os fatores de risco para as doenças cardiovasculares são os mesmos, tanto para adultos quanto para idosos, porém a magnitude do risco relativo associado tende a ser menor após os 65 anos. São considerados fatores de risco: a hipertensão, o colesterol elevado, o tabagismo, o sedentarismo, o abuso do álcool, a situação sócio-econômica, o diabetes, a obesidade e a associação com outras doenças ${ }^{(18)}$.

As estratégias preventivas para as doenças cardiovasculares nos idosos incluem a diminuição da incidência dos fatores de risco, a prevenção primária de episódios clínicos e a implementação de métodos destinados a reduzir os episódios recorrentes, a incapacidade e a morte. Estudo desenvolvido pela Organização Mundial de Saúde ${ }^{(18)}$ conclui que

a prevenção de doenças cardiovasculares nos idosos apresenta desafios diferentes de outras faixas etárias em decorrência da maior variabilidade de condições físicas e mentais.

Assim, a idade por si só, não deve constituir obstáculo para promover a saúde, prevenir e postergar as doenças, a incapacidade e a morte.

As neoplasias malignas corresponderam a 15,8\% dos casos de óbito. O desenvolvimento das neoplasias está direta- mente ligado ao processo de envelhecimento celular pois, com o passar do tempo, diferentes agressões externas vão gerando acúmulos de danos ao DNA das células, possibilitando o desenvolvimento das primeiras células geradoras dos tumores malignos ${ }^{(19)}$. Dessa forma, a idade cada vez mais avançada constitui um fator de risco para o desenvolvimento de neoplasias malignas, o que vem sendo comprovado pelo aumento das taxas de incidência e mortalidade por câncer nas estatísticas nacionais. Os tumores mais freqüentes para os homens, segundo o Instituto Nacional do Câncer, são, em ordem decrescente, os localizados na pele, próstata, estômago, pulmão, boca, esôfago e medula óssea (leucemias) e, para as mulheres, mama, pele, colo uterino, intestino, estômago, pulmão, medula óssea (leucemias), boca e esôfago.

As neoplasias, como morbidade referida, apresentam baixa frequiência, podendo tal fato ser interpretado como um estado desconhecido - por ser tratar de uma doença com alta letalidade e de duração, em geral, relativamente curta ou como um estado não informado ${ }^{(6)}$. Ainda é muito comum, em nossa cultura, que os familiares evitem falar a seus parentes idosos a respeito do seu diagnóstico, quando esse se refere a neoplasias malignas e, muitas vezes, solicitam à equipe de saúde que também o faça. Com isso, muitos idosos acabam desconhecendo o seu diagnóstico e, ao serem indagados, não o citam ${ }^{(19)}$. 
Outro dado interessante a ressaltar é que, embora apresentem maiores taxas de incidência de câncer, as mulheres morrem menos que os homens dessa doença, pois, entre eles, ocorrem cânceres mais letais, como de pulmão e de estômago, fato que chama a atenção para o estabelecimento de programas de detecção precoce de câncer ${ }^{(19)}$.

As doenças do aparelho respiratório apareceram como terceira causa de óbito, correspondendo a $13,2 \%$, com destaque para as doenças pulmonares obstrutivas crônicas (DPOCs). Essas constituem, nos Estados Unidos, a quarta causa de morte entre idosos com idades entre 70 e 90 anos, além de serem importante fator de risco para o desenvolvimento de infecções bacterianas e virais e a causa mais freqüente de hospitalização dos idosos ${ }^{(20)}$. No Brasil, constituem a terceira causa de internação entre os idosos e a segunda entre as doenças respiratórias nessa faixa etária ${ }^{(21)}$.

Estudos epidemiológicos sobre essa doença são ainda limitados, mesmo em países desenvolvidos, dado que sua prevalência é ainda difícil de ser estabelecida, pois não é diagnosticada até que esteja clinicamente aparente e relativamente avançada. Segundo o Global Burden of Disease Study, conduzido pela Organização Mundial de Saúde, citado pelo Instituto Nacional de Saúde ${ }^{(20)}$, a prevalência mundial de DPOC, em 1990, foi estimada em 9,3/1000, em homens, e 7,3/ 1000 , em mulheres. Tais estimativas, no entanto, incluem todas as faixas etárias e subestimam a verdadeira prevalência da doença nos idosos. Sua prevalência é maior nos países onde o consumo de cigarro foi ou é muito comum, evidenciando a correlação da doença com o hábito de fumar.

Embora limitados, os dados disponíveis sobre a morbidade por DPOC indicam que ela aumenta com a idade e é maior entre os homens do que entre as mulheres. É também uma das principais causas de procura por assistência médica, em serviço ambulatorial, hospitalar ou de emergência. Constitui a quarta principal causa de morte no mundo e projeções indicam aumento dessa prevalência nas próximas décadas, em especial entre os idosos. Seu impacto social e econômico é muito importante, pois o mesmo estudo da OMS estimou que a DPOC será a quinta causa principal de perda de Anos de Vida Ajustados à Incapacidade (DisabilityAdjusted Life Year - DALY) no mundo, em 2020, atrás da doença cardíaca isquêmica, da depressão grave, dos acidentes de trânsito e da doença cerebrovascular ${ }^{(20)}$.

Vários estudos epidemiológicos comprovam que a incidência de pneumonia entre os idosos aumenta durante os surtos de gripe e, por essa razão, foi instituída, no Brasil, a vacinação sistemática dos idosos, em 1998. Segundo o DATASUS $^{(21)}$, a pneumonia constitui a segunda causa de internação hospitalar entre os idosos superada apenas pela insuficiência cardíaca. Dentre as doenças respiratórias, supera, inclusive, as DPOCs. As taxas de mortalidade entre os idosos, nos EUA, variam de 15 a $20 \%$, quando adquiridas na comunidade, e de 30 a $40 \%$, quando adquiridas nas institui- ções de longa permanência, representando a sexta causa de morte. A taxa de mortalidade relacionada a essa doença aumentou 59\% em 15 anos, fato atribuído ao envelhecimento da população.

Assim, verifica-se que a pneumonia é uma importante causa de morbidade e mortalidade entre os idosos. O uso de antibióticos profiláticos não a evita, podendo, ao contrário, ocasionar maior resistência bacteriana e tornar o tratamento mais oneroso, prolongado e, às vezes, ineficaz. Assim, a melhor forma de combater essa doença, no idoso, são as medidas preventivas, incluindo a vacinação, além da manutenção de boas condições nutricionais e do tratamento das doenças crônicas concomitantes ${ }^{(20)}$.

As doenças do aparelho digestivo e as doenças endócrinas, nutricionais ou metabólicas apareceram com a mesma proporção de casos (7,9\%). Dentre essas causas de óbito, chamam a atenção algumas condições que poderiam ter sido prevenidas ao invés de culminarem em um evento fatal: úlcera de estômago com hemorragia, desnutrição protéicocalórica e depleção de volume.

Os sangramentos gastrointestinais agudo são muito comum entre os idosos e responsáveis por uma alta taxa de morbi-mortalidade, representando mais de $70 \%$ das internações por doenças do aparelho digestivo. A incidência entre os idosos é dez vezes maior do que entre os adultos jovens e 50\% deles estão relacionados ao uso de antiinflamatórios não esteroidais. A apresentação clínica dos idosos com sangramento pode variar desde a anemia sem sintomas gastrointestinais específicos até as hemorragias maciças. Dentre as doenças do aparelho digestivo, as úlceras gastrointestinais, em $40 \%$ das vezes, e a diverticulose, em $50 \%$, são as maiores responsáveis por tais sangramentos ${ }^{(22-23)}$.

A hemorragia digestiva alta manifesta-se por hematêmese, melena, fezes fétidas, às vezes com sangue vivo e vômitos com aspecto de borra de café e coágulos. A hemorragia digestiva baixa caracteriza-se por fezes sanguinolentas, não fétidas, com exceção daqueles doentes que sangram devido a uma doença intestinal inflamatória. As medidas de ressuscitação incluem expansão volêmica e monitorização hemodinâmica, como em qualquer outra faixa etária. No entanto, é necessário o conhecimento das mudanças nos volumes corporais dos idosos, de forma a não ocasionar situações iatrogênicas que podem ser fatais ${ }^{(22-23)}$.

O diabetes é uma alteração metabólica associada à deficiência parcial ou absoluta da secreção de insulina. Vários estudos epidemiológicos desenvolvidos nos últimos anos têm demonstrado um aumento consistente da incidência e prevalência de diabetes entre os idosos, em especial a do tipo II. A prevalência dessa doença na população brasileira gira em torno de 7,4\% porém, entre os idosos de 60 a 69 anos, esse índice aumenta para $17,4 \%{ }^{(24)}$. 
Embora sejam amplamente divulgados a importância e o impacto do diagnóstico precoce e do bom controle metabólico na evolução e prognóstico da doença, o diabetes é ainda sub-diagnosticado e sub-tratado entre os idosos. As complicações decorrentes dessa doença são responsáveis por incapacidades físicas, como a cegueira, amputações não traumáticas e insuficiência renal, que muitas vezes geram dependência e aumento dos custos com a saúde, tanto com cuidados diretos, quanto com indiretos. Dentre as principais doenças referidas pelos idosos no Estudo SABE, no Município de São Paulo, a única que apresentou decréscimo na velhice avançada foi o diabetes, não por cura e sim, possivelmente, por óbito em decorrência de suas complicações, o que exige maior atenção do sistema de saúde no controle e acompanhamento dessa doença ${ }^{(6)}$.

Ainda procurando entender as razões da morte desses idosos, num segundo momento buscou-se verificar se a causa básica citada na Declaração de Óbito correspondia às doenças referidas pelos idosos quando participaram da primeira fase da coleta de dados do Estudo SABE.

Verificou-se que houve coincidência entre a causa básica do óbito e a doença prévia informada pelo idoso durante a entrevista em $47,4 \%$ dos casos. No entanto, quando houve discordância, ela também foi grande $(39,5 \%)$. Muitos podem ser os fatores envolvidos nessa questão, como o desconhecimento da doença pelo idoso, fato muito comum no caso das neoplasias, por exemplo. Isso é corroborado pelo fato de cinco dos entrevistados $(13,1 \%)$ que foram a óbito não terem citado qualquer doença. Não é muito comum que um indivíduo atinja a velhice sem o desenvolvimento de pelo menos uma doença, geralmente crônica. Assim, é mais provável que ele a desconheça ou a ignore e, portanto, não a informe quando indagado, o que, em última análise, pode proporcionar o não-acompanhamento ou o controle adequado, levando-o a uma condição de risco por agravamento ou à morte.

A qualidade da informação constante na declaração de óbito deve ser considerada como um fator contribuinte para a discordância entre a doença auto-referida e a causa básica do óbito. Estudos mostram que alguns médicos, responsáveis diretos pelo preenchimento da declaração de óbito, ainda não o fazem corretamente, seja porque isso não é ensinado em todas as escolas médicas, seja porque a possibilidade de contribuir para as estatísticas de mortalidade, que é um importante subsídio para a grande maioria dos indicadores de saúde, não é considerado. Desta forma, as causas reais de morte, às vezes, não são colocadas nos atestados, estando mencionadas somente as complicações ou causas terminais ${ }^{(25)}$. Durante a análise realizada, foi observado, por exemplo, que apenas um caso de diabetes constou do atestado de óbito, embora essa doença tenha sido citada por vários idosos.

\section{CONCLUSÕES}

O presente estudo permitiu caracterizar os óbitos dos idosos que faleceram entre a primeira e a segunda fase de coleta de dados do Estudo SABE (Saúde, Bem-Estar e Envelhecimento na América Latina e Caribe).

Dos 38 óbitos ocorridos, a maioria foi do sexo masculino $(55,3 \%)$ e em velhice avançada, ou seja, com idade igual ou superior a 80 anos $(55,3 \%)$. 84,2\% desses idosos viviam com co-residentes e $81,6 \%$ não necessitou de ajuda para responder à primeira fase de coleta de dados do referido estudo, estando, assim, cognitivamente bem.

As principais causas de óbito observadas, segundo a CID-10, foram as doenças do aparelho circulatório $(47,4 \%)$, as neoplasias $(15,8 \%)$, as doenças do aparelho respiratório $(13,2 \%)$, as doenças do aparelho digestivo $(7,9 \%)$, as doenças endócrinas, nutricionais ou metabólicas $(7,9 \%)$ e outras causas $(7,9 \%)$.

Quanto à Declaração de Óbito, verificou-se que, em 47,4\% dos óbitos houve coincidência entre a causa básica declarada e as informações de doenças prévias, obtidas na primeira fase de coleta de dados do presente estudo. Em 39,5\% dessas, no entanto, houve discordância entre as informações, ocasionadas possivelmente por desconhecimento de doenças prévias por parte do idoso, ocorrência de eventos súbitos ou má qualidade da informação obtida nas Declarações de Óbito.

É importante ressaltar as vantagens dos inquéritos domiciliares na complementação das informações de morbimortalidade uma vez que as declarações de óbito ainda não são totalmente representativas da realidade. Haja vista o caso, por exemplo, do diabetes citado por seis dos entrevistados que foram à óbito mas, constante em apenas uma das declarações, em qualquer posição do atestado.

Embora o período de observação tenha sido pequeno (seis meses) o presente estudo encontrou resultados coincidentes com a literatura. Assim, se as doenças crônicas forem precocemente identificadas, adequadamente tratadas e monitoradas, poderá haver redução na mortalidade e nos gastos com a saúde, além de uma melhoria na qualidade de vida dos idosos. 


\section{REFERÊNCIAS}

(1) Passarelli MCG. O processo de envelhecimento em uma perspectiva geriátrica. Mundo Saúde. 1997;21(4):208-12.

(2) Jacob Filho W, Souza RR. Anatomia e fisiologia do envelhecimento. In: Carvalho Filho ET, Papaléo Netto M. Geriatria: fundamentos, clínica e terapêutica. São Paulo: Atheneu; 2000. p. $31-40$.

(3) Veras RP, Lourenço R, Martins CSF, Sanchez MAS, Chaves $\mathrm{PH}$. Novos paradigmas do modelo assistencial no setor saúde: consequiência da explosão populacional dos idosos no Brasil. In: Veras RP. Terceira idade: gestão contemporânea em saúde. Rio de Janeiro: Relume Dumará; 2002. p. 11-79.

(4) Organización Panamericana de la Salud (OPAS) Programa de Coordinación de Investigaciones. División de Salud y Desarrollo Humano. Perfil de las condiciones de salud de los adultos mayores de America Latina y el Caribe. Bol Epidemiol OPS. 1997;18(2):11-3.

(5) Barreto ML, Carmo EH. Situação de saúde da população brasileira: tendências históricas, determinantes e implicações para as políticas de saúde. Inf Epidemiol SUS. 1994;3(3/4):7-34.

(6) Lebrão ML, Laurenti R. Condições de saúde. In: Lebrão ML, Duarte YAO. SABE - Saúde, Bem-Estar e Envelhecimento. O Projeto SABE no Município de São Paulo: uma abordagem inicial. Brasília: Organização Pan-Americana da Saúde; 2003.

(7) Brasil. Ministério da Saúde. DATASUS. Mortalidade - Brasil [online]. http://tabnet.datasus.gov.br/cgi/deftohtm.exe?sim/cnv/ obtuf.def [Acesso em 15 set. 2005].

(8) Lima-Costa MF. Epidemiologia do envelhecimento no Brasil. In: Rouquayrol MZ, Almeida Filho N. Epidemiologia e saúde. Rio de Janeiro: Medsi; 2003. p. 499-513.

(9) Palloni A, Peláez M. Histórico e natureza do estudo. In: Lebrão ML, Duarte YAO. SABE - Saúde, Bem-Estar e Envelhecimento. O Projeto SABE no Município de São Paulo: uma abordagem inicial. Brasília: Organização Pan-Americana da Saúde; 2003. p. $15-32$.

(10) Silva NN. Aspectos metodológicos: processo de amostragem. In: Lebrão ML, Duarte YAO. SABE - Saúde, Bem-Estar e Envelhecimento. O Projeto SABE no Município de São Paulo: uma abordagem inicial. Brasília: Organização Pan-Americana da Saúde; 2003. p. 47-57.

(11) Camarano AA, Kanso S, Mello JL. Como vive o idoso brasileiro. In: Camarano AA, organizador. Os novos idosos brasileiros: muito além dos 60? Rio de Janeiro: IPEA; 2004. p. 25-73.

(12) Santos JLF. Análise de sobrevida sem incapacidades. In: Lebrão ML, Duarte YAO. SABE - Saúde, Bem-Estar e Envelhecimento. O Projeto SABE no Município de São Paulo: uma abordagem inicial. Brasília: Organização Pan-Americana da Saúde; 2003. p. 167-81.

(13) Duarte YAO, Lebrão ML, Lima FD. Contribuição dos arranjos domiciliares para o suprimento de demandas assistenciais dos idosos com comprometimento funcional em São Paulo, Brasil. Rev Panam Salud Publica. 2005;17(5/6):370-8.
(14) Chaimowicz F. A saúde dos idosos brasileiros às vésperas do século XXI: problemas, projeções e alternativas. Rev Saúde Pública. 1997;31(2):184-200.

(15) Ramos LR. Fatores determinantes do envelhecimento saudável em idosos residentes em centro urbano: Projeto Epidoso, São Paulo. Cad Saúde Pública. 2003;19(3):793-7.

(16) Saad PM. Arranjos domiciliares e transferências de apoio informal. In: Lebrão ML, Duarte YAO. SABE - Saúde, BemEstar e Envelhecimento. O Projeto SABE no Município de São Paulo: uma abordagem inicial. Brasília: Organização PanAmericana da Saúde; 2003. p. 201-24.

(17) Organização Mundial de Saúde (OMS). CID-10: classificação estatística internacional de doenças e problemas relacionados à saúde $-10^{\mathrm{a}}$ rev. São Paulo: Centro Colaborador da OMS para a Classificação de Doenças em Português; 1995.

(18) Organización Mundial de la Salud (OMS). Epidemiologia y prevención de las enfermedades cardiovasculares en los ancianos. Ginebra; 1995. (Serie de Informes Técnicos, 853).

(19) Gadelha MIP, Martins RG. Neoplasias no idoso. In: Freitas EV, Py L, Néri AL, Cançado FAX, Gorzoni ML, Rocha SM. Tratado de geriatria e gerontologia. Rio de Janeiro: Guanabara Koogan; 2002. p. 712-7.

(20) Institutos Nacionais da Saúde. Iniciativa global para a doença pulmonar obstrutiva crônica: estratégia global para o diagnóstico, a conduta e a prevenção da doença pulmonar obstrutiva crônica. [s.1.]: AstraZeneca; 1998. (NHLBI/OMS-Resumo executivo).

(21) Brasil. Ministério da Saúde. DATASUS. Morbidade hospitalar do SUS - por local de residência - Brasil [online]. Disponível em: http://tabnet.datasus.gov.br/cgi/tabcgi.exe?sih/cnv/ mruf.def [Acesso em 15 set. 2005].

(22) Moriguti JC. Doenças do aparelho digestório alto. In: Freitas EV, Py L, Néri AL, Cançado FAX, Gorzoni ML, Rocha SM. Tratado de geriatria e gerontologia. Rio de Janeiro: Guanabara Koogan; 2002. p. 381-90.

(23) Lima DCA. Hemorragia digestiva. In: Freitas EV, Py L, Néri AL, Cançado FAX, Gorzoni ML, Rocha SM. Tratado de geriatria e gerontologia. Rio de Janeiro: Guanabara Koogan; 2002. p. 391-8.

(24) Nastri F. Diabetes Mellitus no idoso. In: Freitas EV, Py L, Néri AL, Cançado FAX, Gorzoni ML, Rocha SM. Tratado de geriatria e gerontologia. Rio de Janeiro: Guanabara Koogan; 2002. p. 496-501.

(25) Jorge MHPM, Gotlieb SLD, Laurenti R. O sistema de informações sobre mortalidade: problemas e propostas para o seu enfrentamento. I - Mortes por causas naturais. Rev Bras Epidemiol. 2002;5(2):197-211. 